\title{
LOS DESAFÍOS EN LA CONTINUIDAD DE ATENCIÓN DE PERSONAS VIVIENDO CON VIH EN EL PERÚ DURANTE LA PANDEMIA DE LA COVID-19
}

\author{
Jose L. Paredes@1,a, Rafaella Navarro@1,a, Diego M. Cabrera@3,a, Monica M. Diaz@4, \\ Fernando Mejia(i)1,c, Carlos F. Caceres (iD),d \\ 1 Instituto de Medicina Tropical Alexander von Humboldt, Universidad Peruana Cayetano Heredia, Lima, Perú. \\ 2 Centro de Investigación Interdisciplinaria en Sexualidad, Sida y Sociedad, Lima, Perú. \\ 3 Department of Epidemiology of Microbial Diseases, Yale School of Public Health, New Haven, Connecticut, United States. \\ ${ }^{4}$ University of North Carolina at Chapel Hill, Chapel Hill, North Carolina, United States. \\ a Médico cirujano; ${ }^{\mathrm{b}}$ médico cirujano especialista en Neurología; ${ }^{\mathrm{c}}$ médico cirujano especialista en enfermedades infecciosas \\ y tropicales; ${ }^{\mathrm{d}}$ médico cirujano, magister en Salud Pública.
}

\section{RESUMEN}

La pandemia por la COVID-19 y las medidas restrictivas de distanciamiento social pueden interactuar con la epidemia de VIH de múltiples formas. Existen aproximadamente 87000 personas viviendo con VIH (PVV) en el Perú quienes están en riesgo de contraer la COVID-19; 67000 de ellas que reciben tratamiento antirretroviral (TAR) podrían tener limitaciones en el acceso a sus medicamentos, comprometiendo su adherencia y su salud. Además, el efecto que podría tener la pandemia en la salud mental de PVV en Perú aún no está esclarecido. Este artículo tiene como finalidad describir las implicancias clínicas de la coinfección VIH/SARS-CoV-2; discutir los desafíos en la continuidad de atención de las PVV en el Perú durante la crisis sanitaria por la COVID-19; y comentar las posibles implicancias de las medidas restrictivas sobre la salud mental de las PVV.

Palabras clave: VIH; COVID-19; SARS-CoV-2; Perú, Salud Mental; Continuidad de la Atención al Paciente; Cumplimiento y Adherencia al Tratamiento; SIDA; Infecciones por VIH; Infecciones por Coronavirus (fuente: DeCS BIREME).

\section{CHALLENGES TO THE CONTINUITY OF CARE OF PEOPLE LIVING WITH HIV THROUGHOUT THE COVID-19 CRISIS IN PERU}

Citar como: Paredes JL, Navarro R, Cabrera DM, Diaz MM, Mejia F, Caceres CF. Los desafíos en la continuidad de atención de personas viviendo con VIH en el Perú durante la pandemia de la COVID-19. Rev Peru Med Exp Salud Publica. 2021;38(1):166-70. doi: https://doi.org/10.17843/ rpmesp.2021.381.6471.

Correspondencia: José Luis Paredes; Instituto de Medicina Tropical Alexander von Humboldt, Universidad Peruana Cayetano Heredia. Av. Honorio Delgado 430, San Martín de Porres 15102, Lima, Perú; jose.luis.paredes.s@upch.pe.

Recibido: $16 / 09 / 2020$ Aprobado: 11/11/2020 En línea: 01/02/2021

\begin{abstract}
The COVID-19 pandemic and societal response implemented may interact with the ongoing HIV epidemic in multiple ways. There are approximately 87000 people living with HIV (PLWH) who are at risk of developing COVID-19 in Peru and 67,000 of them are on antiretroviral therapy (ART) and at risk of limitations in their access to ART, compromising their adherence and their health during the pandemic. Finally, the potential effect of the pandemic on the mental health of PLWH is not documented. This opinion aims to: describe the clinical implications of the HIV/SARS-CoV-2 coinfection; discuss the challenges to the continuity of care of PLWH in Peru during the COVID-19 crisis; and comment possible implications that the COVID-19 crisis may pose on the mental health of PLWH.
\end{abstract}

Keywords: HIV; COVID-19; SARS-CoV-2; Peru; mental health; Continuity of Patient Care; Treatment Adherence and Compliance; AIDS; HIV Infections; Coronavirus Infections (source: MeSH NLM).

\section{INTRODUCCIÓN}

La COVID-19 es una enfermedad infecciosa aguda causada por el virus SARS-CoV-2 que fue detectada por primera vez en China en diciembre del 2019 y luego declarada como pandemia $^{(1)}$ por la Organización Mundial de la Salud (OMS). Al 9 de agosto del 2020, hay más de 700000 personas fallecidas en más de 100 países a causa de la COVID-19(2).

En el Perú, el primer caso fue descrito el 6 de marzo del 2020 y cinco días después el gobierno decretó el estado de emergencia sanitaria nacional ${ }^{(3)}$. Diversas acciones se tomaron 
para controlar la trasmisión del virus, como una cuarentena nacional obligatoria desde el 15 de marzo del 2020. A la fecha, Perú es el segundo país más afectado por la COVID-19 en Latinoamérica y a pesar de que la cuarentena nacional ya ha culminado, todavía persisten varias medidas de aislamiento social ${ }^{(2,3)}$.

En el 2019, se estimaba que en el Perú habían 87000 personas viviendo con VIH (PVV), de las cuales 67000 recibían tratamiento antirretroviral (TAR) ${ }^{(4)}$. Las PVV necesitan atención periódica que incluye evaluaciones clínicas, controles laboratoriales y acceso al TAR, lo cual podría verse afectado por la pandemia de la COVID-19 y el distanciamiento social impuesto. Aunque el Ministerio de Salud (MINSA) del Perú ha garantizado la continuidad de la atención sanitaria en las PVV durante la emergencia nacional, la COVID-19 puede afectar las actividades del programa nacional de tratamiento antirretroviral del VIH.

Los objetivos de este articulo son: (1) describir las implicancias clínicas de la coinfección VIH/SARS-CoV-2, (2) discutir los desafíos que la crisis sanitaria plantea para la continuidad de atención de las PVV en el país, y (3) comentar algunas implicaciones para la salud mental de las PVV por las restricciones impuestas para el control de la crisis.

\section{IMPLICANCIAS CLÍNICAS DE LA COINFECCIÓN VIH/SARS-COV-2}

En el 2019 había 38 millones de PVV en el mundo, entre las cuales el riesgo de COVID-19 y su presentación clínica aún no están esclarecidos. Al respecto se han publicado dos revisiones sistemáticas: una que incluyó ocho estudios (70 PVV) ${ }^{(5)} \mathrm{y}$ otra que incluyó 25 estudios (252 PVV) ${ }^{(6)}$. En esta última, la edad media fue de 52,7 años; el 89\% fueron varones; el 98\% recibía TAR y el $87 \%$ tenía un conteo de CD4 >200. Asimismo, el 59\% tenía por lo menos una comorbilidad - principalmente hipertensión arterial y obesidad/hiperlipidemia- y el 65\% estaban hospitalizados. El 17\% requirió manejo en la unidad de cuidados intensivos y el $14,3 \%$ falleció ${ }^{(6)}$, cifra que no parece ser mayor a la reportada en pacientes hospitalizados ${ }^{(7)}$.

Los autores concluyen que las PVV están expuestas al riesgo de contraer la COVID-19 y presentan patrones de progresión similares a los de personas VIH negativas; y que las comorbilidades múltiples y la mayor edad en las PVV parecen asociarse a morbilidad severa y mortalidad por la COVID-19. Por ello, es importante afrontar las comorbilidades de las PVV, asegurarse de la continuidad de su tratamiento, y considerarlos como una población vulnerable. La escasez de datos sobre la coinfección, pese al gran número actual de PVV en el mundo, sugiere que muchas coinfecciones no estarían siendo diagnosticadas (posiblemente porque no se pregunta por la condición de VIH o no se revela su presencia), o no se les estaría consignando en las historias clínicas.
Se requiere identificar y estudiar mayor número de coinfecciones VIH/SARS-CoV-2 para entender su presentación clínica e implicancias.

\section{DESAFÍOS EN LA CONTINUIDAD DE ATENCIÓN DE PERSONAS VIVIENDO CON VIH}

En el 2014, el Programa Conjunto de las Naciones Unidas sobre el VIH/SIDA (ONUSIDA) lanzó las «metas 90-90-90» ${ }^{(8)}$ para el 2020, es decir, que el $90 \%$ de todas las PVV conozcan su estado serológico, que el 90\% de las PVV diagnosticadas reciban TAR, y que $90 \%$ de las que reciben tratamiento logren supresión viral ${ }^{(8)}$, para eliminar la transmisión en el 2030.

En el mundo se han logrado avances importantes para las «metas 90-90-90» ${ }^{\left({ }^{8}\right)}$. En el Perú, en el 2014, de las 72000 PVV estimadas, el 64\% había sido diagnosticado, el 46\% estaban en TAR y el 36\% había logrado la supresión viral ${ }^{(9)}$. Para el 2018, una nueva medición reportó mejores cifras: el $78 \%$ de las PVV conocían su diagnóstico, el 76\% estaban en TAR y el $65 \%$ habían alcanzado la supresión viral ${ }^{(10)}$. A pesar de la evidente mejoría para lograr las «metas 90-90-90», en el 2020 todavía quedaba bastante trabajo que realizar.

Pese a que el MINSA garantizó la distribución continua de la TAR a las PVV durante la crisis sanitaria, los desafíos ya existentes para cumplir los objetivos de ONUSIDA en el Perú podrían haberse incrementado, debido a la reasignación de los recursos en salud, menor disponibilidad de laboratorios, licencias otorgadas al personal de salud con comorbilidades, alteración del abastecimiento de TAR a los establecimientos, la suspensión y/o restricción de muchos servicios regulares de salud, y el temor de los mismos usuarios a acercarse a los establecimientos ${ }^{(11)}$.

En el Perú, hasta el 2 de octubre del 2020 se notificaron 1494 nuevos casos de infección por VIH, lo que representa el $18,2 \%$ de los casos notificados en el $2019^{(12)}$. Esto podría significar que un importante número de PVV no habrían sido diagnosticadas ni vinculadas a los servicios de tratamiento. En este contexto, las pruebas autoadministradas de VIH, recomendadas por la OMS, resultan una opción prometedora para poder aumentar el tamizaje del VIH durante la pandemia. Consisten en que una persona se tome una muestra (de saliva o sangre), se realice la prueba e interprete el resultado. Estas pruebas permiten abordar barreras importantes como los largos tiempos de espera en los centros de salud, y el estigma percibido ${ }^{(13)}$; asimismo, eliminarían la necesidad de visitar los establecimientos de salud durante la crisis sanitaria.

En Perú un estudio transversal reportó que el 82\% de los hombres que tienen sexo con hombres y mujeres transgénero están dispuestos a realizarse una prueba autoadministrada de VIH cuatro veces al año, como lo recomienda la OMS 
para poblaciones de riesgo ${ }^{(14)}$. Por lo tanto, promover las pruebas autoadministradas de VIH podría ser una alternativa útil para abordar la primera meta de ONUSIDA durante este período.

Para lograr el segundo y tercer objetivo de las «metas 90-90-90», es fundamental lograr una adherencia óptima al TAR ${ }^{(8)}$. Por lo tanto, se debe asegurar un suministro por períodos más largos - por ejemplo, de tres a seis mesespara reducir el número de visitas, limitando la exposición al virus; y garantizando la recepción del tratamiento ${ }^{(15)}$. En la India, la entrega del TAR a domicilio ha permitido un suministro adecuado de medicamentos contra el VIH ${ }^{(16)}$ y en Tailandia, los hospitales dispensan el TAR en periodos de tres a seis meses ${ }^{(17)}$. En contraposición, en Kigali, Ruanda, solo el 48\% de las PVV habían asistido a sus citas para el recojo de TAR durante la cuarentena ${ }^{(18)}$.

El efecto de la crisis sanitaria sobre el recojo de medicamentos y la adherencia al TAR en PVV aún no ha sido completamente valorado en el Perú, aunque hay indicios de que las PVV estarían experimentando dificultades para ser adherentes al TAR ${ }^{(19)}$. A pesar de que las guías internacionales recomiendan el suministro del tratamiento para 90 días en el contexto de la COVID-19 ${ }^{(16,17)}$, un hospital de referencia en Lima experimentó escasez de TAR (zidovudina, lamivudina, lopinavir, ritonavir y abacavir), lo que provocó cambios temporales en el régimen de tratamiento en algunas PVV. Por otro lado, debido al estigma del VIH, algunas PVV prefieren recibir su TAR en centros de salud lejos de sus comunidades, lo cual podría ser complicado ahora, ya que el transporte público se ha visto restringido.

El inicio temprano del TAR es fundamental para garantizar resultados óptimos en términos individuales - supresión viral, mayor recuento de células CD4, menos enfermedades oportunistas - y de beneficio social - reducción de la transmisión del VIH-. Este proceso podría ser difícil de lograr durante la crisis sanitaria por la suspensión de las consultas ambulatorias, ya que las PVV recientemente diagnosticadas deben ser evaluadas por un médico, un psicólogo y un trabajador social antes de iniciar el TAR en el Perú (20).

La implementación de la telemedicina sería óptima para la continuidad de la atención de las PVV durante la pandemia, pues facilitaría el inicio y el seguimiento del tratamiento. La telemedicina puede prevenir los problemas que las PVV sufrieron durante la pandemia de la COVID-19, tales como las experiencias estigmatizantes en los medios de transporte público, cuando tuvieron que movilizarse con permisos de circulación que indicaban su estado de ser VIH positivo, y minimizar su exposición al SARS-CoV-2. Sin embargo, las barreras para la implementación de la telemedicina en el Perú incluyen la falta de acceso a Internet y la falta de equipos de teleconferencia en muchos centros de salud.

Finalmente, es importante resaltar que las medidas de aislamiento social y la pandemia podrían estar afectando la atención sanitaria de las PVV en las zonas rurales. Estas comunidades están dispersas y usualmente las PVV deben desplazarse a los centros de salud de tercer nivel para recibir su TAR. Durante la pandemia, las restricciones en el transporte podrían haber afectado el acceso a los centros de salud y el recojo del TAR; asimismo, no se sabe si ha habido desabastecimiento de fármacos antirretrovirales en centros de salud de zonas rurales. El control del VIH está amenazado por la COVID-19 en el Perú y en todo el mundo, y los expertos estiman que se producirían 500000 muertes debido a la interrupción en la atención sanitaria de PPV ${ }^{(21)}$. La elaboración de estrategias para mejorar el diagnóstico del VIH, asegurar el suministro hospitalario y la entrega del TAR, y el acceso a la atención medica es fundamental para evitar una sobrecarga de los hospitales y un aumento de la morbimortalidad de las PVV.

\section{IMPLICANCIAS DE LA COVID-19 EN LA SALUD MENTAL DE LAS PERSONAS VIVIENDO CON VIH}

La pandemia de la COVID-19 podría tener un impacto negativo en la salud mental, especialmente en personas con enfermedades preexistentes como el VIH ${ }^{(22)}$. Antes de la pandemia, las PVV en Perú tenían una alta carga de afecciones de salud mental, incluida la depresión (entre $48 \%$ y $68 \%)^{(23,24)}$ y la percepción de estigma y rechazo (más del $50 \%)^{(25)}$. En el contexto de la COVID-19, debido al miedo de contraer el virus, la soledad, y la incertidumbre económica, entre otros factores, se ha visto un aumento en la prevalencia de depresión en la población general ${ }^{(22)}$, lo cual también afectaría a las PVV. Asimismo, una consecuencia importante de la crisis sanitaria es un incremento en la percepción de soledad y aislamiento en la población general. Considerando la alta prevalencia de estigma y rechazo — que son factores asociados a un mayor aislamiento- en las PVV en Perú, el aislamiento de esta población podría aumentar, afectando su salud mental.

Las PVV - especialmente las mujeres - experimentan tasas más altas de violencia doméstica en comparación con la población general ${ }^{(26)}$. Considerando que durante la pandemia de la COVID-19, la violencia doméstica ha aumentado a nivel mundial ${ }^{(22)}$ las PVV podrían estar en mayor riesgo. Por otro lado, el $71 \%$ de la población económicamente activa en Perú, incluidas las PVV, tienen trabajos independientes e informales, sin salarios fijos. Estas personas podrían haber perdido sus ingresos durante la cuarentena, lo cual incrementaría sus niveles de ansiedad y depresión. Finalmente, al buscar nuevas fuentes de ingresos, su exposición al SARS-CoV-2 también aumentaría $^{(27)}$.

La pandemia y las medidas de aislamiento social implementadas por el gobierno peruano podrían impactar directamente en la salud mental de las PVV, aumentar la prevalencia 
de depresión, de aislamiento social y potencialmente de violencia doméstica. Además, el posible aumento de la depresión en las PVV podría afectar su salud considerando que las PVV que experimentan síntomas de depresión tienen 42\% menos probabilidad de tener una buena adherencia al TAR ${ }^{(28)}$. Durante las restricciones, la telemedicina podría mejorar la detección y el tratamiento de los trastornos de salud mental en esta población, con un mayor énfasis en la depresión, la percepción de aislamiento social, la violencia doméstica y su impacto en la adherencia al TAR. La OMS apoya el uso de la telemedicina para ayudar a los pacientes con problemas de salud mental durante esta crisis sanitaria, y alienta a los proveedores de salud a detectar activamente estos pacientes, ofrecerles apoyo y tratamiento médico ${ }^{(29)}$.

\section{CONCLUSIONES}

La crisis sanitaria por la COVID-19 y las medidas impuestas para su control pueden representar un desafío considerable para las PVV, especialmente en países de ingresos bajos y medios como el Perú. Es necesario investigar más sobre las implicancias de la coinfección VIH/SARS-CoV-2 en el país y generar evidencia sobre el efecto de la pandemia y de las medidas de aislamiento social en la adherencia al TAR y en la atención sanitaria de las PVV. Asimismo, se deben realizar esfuerzos para asegurar el diagnóstico, vinculación y entrega del TAR, y las evaluaciones médicas a las PVV, a través de sistemas de telemedicina. Finalmente, el alto riesgo de depresión, aislamiento social y violencia doméstica que puede ocurrir en las PVV requiere la implementación de estrategias de protección de la salud mental. Será solo con creatividad y con el compromiso de los sistemas de salud que los efectos de la crisis de la COVID-19 en las PVV se podrán prevenir o mitigar en el Perú y en todo el mundo.

Contribuciones de los autores: JLP, RN, DMC, MMD estuvieron involucrados en la revisión de la literatura y escritura del artículo. FM y CFC estuvieron involucrados en el planteamiento del articulo y la revisión crítica de este. Todos los autores verificaron la versión final del artículo.

Financiamiento: Ninguno.

Conflicto de interés: Ninguno.

\section{REFERENCIAS BIBLIOGRÁFICAS}

1. World Health Organization. Cumulative COVID-19 cases reported by countries and territories in the Americas, 2020 [Internet]. USA: WHO; 2020. [citado el 08 de agosto de 2020]. Disponible en: https:// who.maps.arcgis.com/apps/webappviewer/index.html?id=2203b04c3a5f486685a15482a0d97a87\&extent=-17277700.8881\%2C-1043174.5225 \%2C-1770156.5897\%2C6979655.9663\%2C102100.

2. Johns Hopkins University. COVID-19 Dashboard by the Center for Systems Science and Engineering (CSSE) at Johns Hopkins University (JHU), 2020 [Internet]. USA: JHU; 2020. [citado el 08 de agosto de 2020]. Disponible en: https://coronavirus.jhu.edu/map.html.

3. Decreto Supremo que declara Estado de Emergencia Nacional por las graves circunstancias que afectan la vida de la Nación a consecuencia del brote del COVID-19 [Internet]. Diario El Peruano. [citado el 08 de agosto de 2020]. Disponible en: https://busquedas.elperuano.pe/normaslegales/ decreto-supremo-que-declara-estado-de-emergencia-nacional-po-decreto-supremo-n-044-2020-pcm-1864948-2/.

4. Joint United Nations Programme on HIV/AIDS (UNAIDS) [Internet]. USA: UNAIDS; 2020 [citado el 08 de agosto de 2020]. Disponible en: https://www.unaids.org/es/regionscountries/countries/peru.

5. Cooper T, Woodward B, Alom S, Harky A. Coronavirus disease 2019 (COVID-19) outcomes in HIV/AIDS patients: a systematic review. HIV Med. 2020; 21: 567-7. doi: 10.1111/hiv.12911.

6. Mirzaei H, McFarland W, Karamouzian M, Sharifi H. COVID-19 Among People Living with HIV: A Systematic Review. AIDS Behav. Epub of print 30 July 2020. doi: 10.1007/s10461-020-02983-2.

7. Tian W, Jiang W, Yao J, Nicholson C, Li R, Sigurslid H, et al. Predictors of mortality in hospitalized COVID-19 patients: A systematic review and meta-analysis. J Med Virol. 2020; 92:1875-83. doi: 10.1002/jmv.26050.

8. Joint United Nations Programme on HIV/AIDS (UNAIDS). [Internet]. USA: UNAIDS; 2020 [citado el 08 de agosto de 2020]. Disponible en: https://www.unaids.org/sites/default/files/media_asset/JC2686_WAD2014report_en.pdf.

9. Garcia-Fernandez L, Novoa R, Huaman B, Benites C. Continuo de la atención de personas que viven con VIH y brechas para el logro de las metas 90-90-90 en Perú. Rev Peru Med Exp Salud Publica. 2018; 35:491-6. doi: 10.17843/rpmesp.2018.353.3853.

10. Cáceres C. Estudio sobre el Continuo de Atención de las Personas con VIH Lima: Centro de Investigación Interdisciplinaria en sexualidad, SIDA y Sociedad; 2019.

11. Diario Oficial 'El Peruano'. Suspenden consultas externas en hospitales del Minsa y Essalud. [Internet]. Lima: El Peruano; 2020 [citado el 08 de agosto de 2020]. Disponible en: https://elperuano.pe/noticia/93120-suspenden-consultas-externas-en-hospitales-del-minsa-y-essalud.

12. Ministerio de Salud. Sala situacional VIH/SIDA, 2020 [Internet]. Lima: Centro Nacional de Epidemiologia, Prevención y Control de Enfermedades, MINSA; 2020 [citado el 2 de octubre de 2020]. Disponible en: https:// www.dge.gob.pe/vih/sala/index/fig1_Curvavih/2213.

13. Johnson CC, Kennedy C, Fonner V, Siegfried N, Figueroa C, Dalal S, et al. Examining the effects of HIV self-testing compared to standard HIV testing services: a systematic review and meta-analysis. J Int AIDS Soc. 2017;20(1):21594. doi: 10.7448/IAS.20.1.21594.

14. Bustamante MJ, Konda KA, Joseph Davey D, Leon SR, Calvo GM, Salvatierra J, et al. HIV self-testing in Peru: questionable availability, high acceptability but potential low linkage to care among men who have sex with men and transgender women. Int J STD AIDS. 2017; 28: 133-7.doi: $10.1177 / 0956462416630674$.

15. International AIDS Society. COVID-19 and HIV: What you need to know [Internet]. USA: IAS; 2020 [citado el 08 de agosto de 2020]. Disponible en: https://www.iasociety.org/covid-19-hiv.

16. Joint United Nations Programme on HIV/AIDS (UNAIDS). Mitigating the impact of the COVID-19 pandemic among people living with HIV in India, [Internet]. USA: UNAIDS; 2020 [citado el 08 de agosto de 2020]. Disponible en: https://www.unaids.org/en/resources/presscentre/ featurestories/2020/may/20200506_india (2020).

17. Joint United Nations Programme on HIV/AIDS (UNAIDS). Thai hospitals to provide three- to six-month supplies of antiretroviral therapy, [Internet]. USA: UNAIDS; 2020 [citado el 08 de agosto de 2020]. Disponible en: https://www.unaids.org/en/resources/presscentre/featurestories/2020/ march/20200325_thailand. 
18. Pierre G, Uwineza A, Dzinamarira T. Attendance to HIV Antiretroviral Collection Clinic Appointments During COVID-19 Lockdown. A Single Center Study in Kigali, Rwanda. AIDS Behav. 2020;24(12):3299-3301. doi: 10.1007/s10461-020-02956-5.

19. El Comercio. Las víctimas colaterales del COVID: vivir con VIH en medio de la pandemia, entre la falta de medicinas y el miedo [Internet]. Peru: El Comercio; 2020 [citado el 08 de agosto de 2020]. Disponible en: https:// elcomercio.pe/tecnologia/ciencias/coronavirus-vih-sida-las-victimas-colaterales-del-covid-vivir-con-vih-en-medio-de-la-pandemia-entre-la-falta-de-medicinas-y-el-miedo-noticia/?ref=ecr.

20. Ministerio de salud del Perú. Norma técnica de salud de atención integral del adulto con infección por el virus de la inmunodeficiencia humana (VIH) [Internet]. Lima: MINSA; 2020 [citado el 08 de agosto de 2020]. Disponible en: http://bvs.minsa.gob.pe/local/MINSA/4479.pdf.

21. International Association of providers of AIDS care. Global Experts Warn of COVID-19-Related HIV Service Disruptions [Internet]. USA: Int. Assoc. Provid. AIDS Care; 2020 [citado el 08 de agosto de 2020]. Disponible en: https://www.iapac.org/2020/07/15/global-experts-warn-covid-19-hiv-service-disruptions/.

22. Holmes EA, O’Connor RC, Perry VH, Tracey I, Wessely S, Arseneault L et al. Multidisciplinary research priorities for the COVID-19 pandemic: a call for action for mental health science. The Lancet Psychiatry 2020; 7: 547-560. doi: 10.1016/S2215-0366(20)30168-1.

23. Shin S, Muñoz M, Espiritu B, Zeladita J, Sanchez E, Callacna M, et al. Psychosocial Impact of Poverty on Antiretroviral Nonadherence Among HIV-TB Coinfected Patients in Lima, Peru. J Int Assoc Physicians AIDS Care 2008; 7: 74-81. doi: 10.1177/1545109708315326.
24. Wu DY, Munoz M, Espiritu B, Zeladita J, Sanchez E, Callacna M, et al. Burden of Depression Among Impoverished HIV-Positive Women in Peru. J Acquir Immune Defic Syndr. 2008;48(4):500-4. doi: 10.1097/ QAI.0b013e31817dc3e9.

25. Zafra-Tanaka JH, Ticona-Chavez E. Estigma relacionado a VIH/SIDA asociado con adherencia al tratamiento antirretroviral en pacientes de un hospital de Lima, Perú 2014. Rev Peru Med Exp Salud Publica. 2016; 33: 625. doi: 10.17843/rpmesp.2016.334.2544.

26. Li Y, Marshall CM, Rees HC, Nunez A, Ezeanolue EE, Ehiri J. Intimate partner violence and HIV infection among women: a systematic review and meta-analysis. J Int AIDS Soc. 2014;17(1):18845. doi: 10.7448/ IAS.17.1.18845.

27. BBC news mundo. Cuarentena en Perú: 5 factores que explican por qué las medidas de confinamiento no impiden que sea el segundo país de América Latina con más casos de covid-19. [Internet]. USA: BBC; 2020 [citado el 08 de agosto de 2020]. Disponible en: https://www.bbc. com/mundo/noticias-america-latina-52748764.

28. Uthman OA, Magidson JF, Safren SA, Nachega JB. Depression and Adherence to Antiretroviral Therapy in Low-, Middle- and High-Income Countries: A Systematic Review and Meta-Analysis. Curr HIV/AIDS Rep. 2014; 11:291-307. doi: 10.1007/s11904-014-0220-1.

29. World Health Organization. Addressing Human Rights as Key to the COVID-19 Response, [Internet]. USA: WHO; 2020 [citado el 08 de agosto de 2020]. Disponible en: https://www.who.int/publications/i/ item/addressing-human-rights-as-key-to-the-covid-19-response. 